\title{
Morphogenetic Effects of Mutations at the $A$ and $B$ Incompatibility Factors in Coprinus cinereus
}

\author{
By S. SWAMY, ISAO UNO AND TATSUO ISHIKAWA* \\ Institute of Applied Microbiology, University of Tokyo, Bunkyo-ku, Tokyo 113, Japan
}

(Received 15 May 1984 ; revised 14 August 1984)

\begin{abstract}
Mutated $A$ and $B$ incompatibility factors of Coprinus cinereus ( $A m u t$ and $B m u t$ ) were recovered from fruit bodies produced on common- $A$ and common- $B$ heterokaryons, respectively, following mutagenesis. The Amut hyphal cells were either uninucleate or binucleate and had pseudoclamps irregularly scattered along the hyphae. The Bmut hyphal cells were predominantly uninucleate and had no clamp structures. Amut Bmut double mutants constructed from these Amut and Bmut strains were predominantly binucleate, had true clamps, and gave rise to fertile fruit bodies indistinguishable from those of wild-type dikaryons. Although these Amut Bmut strains resembled in most respects a normal dikaryon, they produced abundant oidiophores and oidia like the monokaryons. The oidia were uninucleate, and possessed the potential to grow into fertile homokaryons with the above characteristics.
\end{abstract}

\section{INTRODUCTION}

In many fungi where sexual dimorphism is absent, sexual reproduction is primarily subjected to genetic control. In the tetrapolar Hymenomycetes typified by Schizophyllum commune and Coprinus cinereus, the genetic control involved operates in the morphogenetic sequence leading to the formation of a fruit body (Raper, 1966; Casselton, 1978). Karyogamy and meiosis, the underlying features of sexual reproduction, take place in the fruit body. The ability of two heterothallic strains to mate successfully is governed by an incompatibility control system composed of two multiallelic factors, $A$ and $B$, in the haploid genome (Raper \& San Antonio, 1954; Swiezynski \& Day, 1960a; Raper, 1966; Koltin, 1978). Both factors possess distinct regulatory functions in the sequence of events leading to sexual morphogenesis (Swiezynski \& Day, 1960a,b; Raper, 1966; Casselton, 1978).

Mutants of $A$ and $B$ factors (Amut and Bmut) have been isolated in Schizophyllum and Coprinus and an analysis of these has shown two interesting features (Parag, 1962; Day, 1963; Raper et al., 1965; Koltin et al., 1972; Haylock et al., 1980). Firstly, a majority of the mutations led to the conversion of self-incompatible strains to self-compatible ones. Secondly, the phenotypes of most of these mutants revealed constitutive expression of events regulated by the mutated factor.

Confirmation of the regulatory role played by the incompatibility factors in sexual reproduction was obtained in Schizophyllum with the isolation of Amut Bmut strains which contained the two mutated factors in the same haploid nucleus (Raper et al., 1965; Raper \& Raper, 1966; Koltin, 1970). These mutant strains not only resembled dikaryons in various morphological characteristics but were also sexually fertile. More recently, Amut Bmut strains were recovered in Coprinus as well, however, sexual morphogenesis in these was incomplete as they failed to produce mature fruit bodies (Haylock et al., 1980).

The present paper describes the isolation and characterization of $A m u t$ and $B m u t$ strains in $C$. cinereus from which Amut Bmut double mutant strains were constructed. These Amut Bmut strains exhibited several features characteristic of the dikaryon including the presence of true

Abbreviation: NTG, $N$-methyl- $N^{\prime}$-nitro- $N$-nitrosoguanidine. 
clamps and production of mature fruit bodies, but they retained a few features typically associated with monokaryons such as production of abundant oidia.

\section{METHODS}

Strains. The following wild-type and auxotrophic mutant strains of Coprinus cinereus (formerly designated as $C$. macrorhizus Rea f. microsporus Hongo) were used: $A 2 B 2$ ad-2, A2B2 chol-1, A2B2 paba-1, A2B8 paba-1, A7B2 ad-1, $A 7 B 7$ arg-2, $A 8 B 7 \mathrm{ad}-1$, and $A 8 B 8$. Wild-type stocks with appropriate incompatibility factors were kindly provided by Professor T. Takemaru, Okayama University, Okayama, Japan. Heterokaryotic and dikaryotic strains used in this study were obtained by hyphal fusion of two of the above strains in appropriate combinations.

Media and cultivation. Heterokaryons were constructed on Fries minimal medium (MM medium) (Fries, 1953). For fruiting, cultures were prepared on or transferred to a malt/yeast medium (MY medium) (Uno \& Ishikawa,

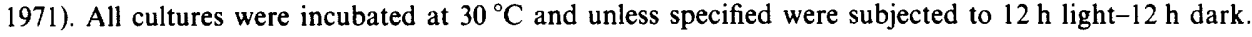

Isolation of incompatibility factor mutants. NTG was used as the mutagen. For the isolation of $A$ - and $B$-factor mutants, common- $A$ and common- $B$ heterokaryons were established by mating monokaryotic auxotrophs with complementary nutritional requirements on $\mathrm{MM}$ medium containing 50 and $60 \mu \mathrm{g} \mathrm{NTG} \mathrm{ml}^{-1}$, respectively, and incubating these plates at $30^{\circ} \mathrm{C}$ in the dark for 3-5 d. Once the heterokaryon was established, the cultures were transferred to MY medium and incubated under the conditions specified above to test for fruiting. Basidiospores produced on the fruit bodies were tested for the segregation of $A$ - or $B$-factor mutants.

Fluorescence microscopy. Samples were placed on a glass slide, and a drop of staining solution as described by Kuroiwa et al. (1981) was added immediately containing $10 \mu \mathrm{g}$ propidium iodide $\mathrm{ml}^{-1}$ (Sigma) and $500 \mu \mathrm{g}$ ribonuclease $\mathrm{A} \mathrm{ml}^{-1}$ (Sigma). The materials were then squashed gently under a coverslip. Propidium iodide staining and ribonuclease treatment were done simultaneously at $20^{\circ} \mathrm{C}$ for $30 \mathrm{~min}$. Observation of fluorescence was done with a UVFL 100 objective using a UV excitation filter $(545 \mathrm{~nm})$ in combination with a $610 \mathrm{~nm}$ suppression filter. Photographs were taken on $35 \mathrm{~mm}$ Fuji 400 film.

\section{RESULTS AND DISCUSSION}

\section{Isolation and characterization of $A$ - and $B$-factor mutants}

Common- $A$ and common- $B$ heterokaryons of the compositions $A 2 B 2$ chol- $1+A 2 B 8$ paba- 1 and $A 2 B 2$ ad-2 $+A 7 B 2$ ad-1, respectively, were treated with NTG at $30^{\circ} \mathrm{C}$ for $3-5 \mathrm{~d}$ and then incubated at $30^{\circ} \mathrm{C}$ for $15-30 \mathrm{~d}$ under the conditions specified to test for fruiting. Four out of 100 common- $A$ heterokaryon cultures and three out of 700 common- $B$ heterokaryon cultures thus treated produced fruit bodies. Basidiospores which formed on the fruit bodies of these fertile cultures were subjected to random spore analysis. Analysis of $A$ - and $B$-factor specificities of the isolated progenies revealed an approximately $1: 1$ segregation of the relevant parental factors and mutant $A$ or $B$ factors which were cross-compatible with the original parental alleles. The mutated $A 2$ and $B 2$ factors identified in these crosses were designated Amut and Bmut, respectively.

To confirm whether the mutations had occurred within the respective incompatibility factor loci or not, the progenies of crosses involving Amut $\times A 8$, and $B m u t \times B 7$ were screened for the presence of parental $A 2$ and $B 2$ factors, respectively (Table 1). None of the crosses revealed the presence of the original parental factors, but an approximately $1: 1$ segregation of the mutated

Table 1. Crosses showing the loss of original incompatibility factors as a result of mutation in the $A$ and $B$ factors

\begin{tabular}{|c|c|c|c|c|c|c|c|c|c|}
\hline \multirow[b]{2}{*}{ Cross* } & \multirow{2}{*}{$\begin{array}{c}\text { Total no. of } \\
\text { single spore } \\
\text { isolates } \\
\text { analysed }\end{array}$} & \multicolumn{4}{|c|}{$\begin{array}{l}\text { Segregation of } \\
A \text { factors }\end{array}$} & \multicolumn{4}{|c|}{$\begin{array}{c}\text { Segregation of } \\
B \text { factors }\end{array}$} \\
\hline & & $A 2$ & Amut & $A 7$ & $A 8$ & $B 2$ & Bmut & $B 7$ & $B 8$ \\
\hline Amut $B 8 \times A 8 B 7$ & 97 & 0 & 47 & - & 50 & - & - & 53 & 44 \\
\hline Amut $B 2 \times A 8 B 7$ & 172 & 0 & 82 & - & 90 & 91 & - & 81 & - \\
\hline$A 7$ Bmut $\times A 8 B 7$ & 383 & - & - & 190 & 193 & 0 & 188 & 195 & - \\
\hline$A 2 B m u t \times A 8 B 7$ & 112 & 58 & - & - & 54 & 0 & 51 & 61 & - \\
\hline
\end{tabular}

* The Amut and Bmut strains were selected from those recovered from basidiospores formed on the fruit bodies of the original mutant cultures. 
Table 2. Distribution of nuclei, clamp cell characteristics and number of oidia in wild-type and mutant strains

\begin{tabular}{|c|c|c|c|c|c|c|c|}
\hline \multirow[b]{2}{*}{ Strain } & \multicolumn{3}{|c|}{$\begin{array}{l}\text { No. of nuclei per } \\
\text { hyphal cell }\end{array}$} & \multicolumn{3}{|c|}{$\begin{array}{l}\text { Proportion and type of } \\
\text { clamp cells present }\end{array}$} & \multirow{2}{*}{$\begin{array}{l}\text { No. of oidia } \\
\text { per culture* }\end{array}$} \\
\hline & 1 & 2 & 3 & None & Pseudoclamp & True clamp & \\
\hline $\begin{array}{l}\text { Monokaryon } \\
\quad(A 7 \text { B7 arg-2) }\end{array}$ & 97 & 0 & 0 & 97 & 0 & 0 & $6.4 \times 10^{7}$ \\
\hline $\begin{array}{l}\text { Dikaryon } \\
\quad(A 2 B 2+A 8 B 8)\end{array}$ & 0 & 107 & 0 & 0 & 0 & 99 & $3.0 \times 10^{4}$ \\
\hline Amut B8 & 49 & 37 & 5 & 48 & 39 & 5 & $5.6 \times 10^{7}$ \\
\hline$A 7$ Bmut & 113 & 1 & 0 & 114 & 0 & 0 & $9.2 \times 10^{6}$ \\
\hline Amut Bmut-1 & 11 & 112 & 1 & 2 & 21 & 101 & $8.0 \times 10^{6}$ \\
\hline
\end{tabular}

* Suspensions of oidia were prepared from 6-day-old cultures grown in MY media contained in $100 \mathrm{ml}$ Erlenmeyer flasks.

factors and the relevant wild-type factors was observed. These results indicated that the mutations had either occurred within the respective incompatibility factor loci or at sites very closely linked to them.

The phenotypic consequences of these mutations were examined with respect to nuclear distribution and clamp characters. In Amut strains a majority of the cells were either uninucleate or binucleate although a few cells containing three nuclei were found among those examined (Table 2). The occurrence of clamp structures in these strains was extremely variable with about half the cells examined having no clamps. The remainder of the cells had pseudoclamps, which were scattered irregularly along the hyphae, and occasionally true clamps (Table 2). These features were essentially similar to those exhibited by the Amut strains isolated by Day (1963) in Coprinus and Raper et al. (1965) in Schizophyllum.

The hyphal cells of Bmut strains that were examined were predominantly uninucleate and showed no clamp structures (Table 2). Our observations thus confirm the findings of Haylock et $a l$. (1980) that the $B m u t$ cells were predominantly uninucleate and mutation in the $B$ factor had not been accompanied by constitutive expression of septum disruption. These observations in the Bmut strains of Coprinus were in contrast to those found in Schizophyllum where the Bmut cells were found to be either uninucleate or binucleate with a significant number of cells containing three or more nuclei per cell (Raper \& Raper, 1966).

\section{Recovery and characterization of $A$ - and $B$-factor double mutants}

The Amut and Bmut strains isolated as described above were crossed with each other and the resulting heterokaryon gave rise to fertile fruit bodies. Analysis of single spore isolates from these fruit bodies revealed the segregation of four classes of progeny: wild-type monokaryons, Amut, Bmut and Amut Bmut double mutants in approximately equal frequency (Table 3). The Amut Bmut double mutants could be visually recognized by the formation of fertile fruit bodies, the morphology of which strikingly resembled that of dikaryotic fruit bodies (Fig. 1).

To find out whether these strains were heterokaryotic in nature, single spore isolations of basidiospores formed on Amut Bmut fruit bodies were carried out. All of the isolates grew into colonies which were dikaryotic in appearance and self-fertile, indicating that the two mutations were located in the same haploid nucleus. The Amut Bmut strains were compatible with all available monokaryotic tester strains. An analysis of a few of their progenies revealed as expected the segregation of wild-type monokaryons, Amut, Bmut and Amut Bmut homokaryons in approximately equal frequency (Table 3).

Examination of the phenotype of the hyphae of Amut Bmut strains revealed several features characteristic of the dikaryon, including the predominance of binucleate cells and true clamp connections (Table 2). In these strains where both the $A$ - and $B$-dependent sequences were 'on', the percentage of clamp cells formed was much higher as compared to the Amut strains (Table 2 ), indicating that the extent to which clamp cells are formed might be related to the sequential 

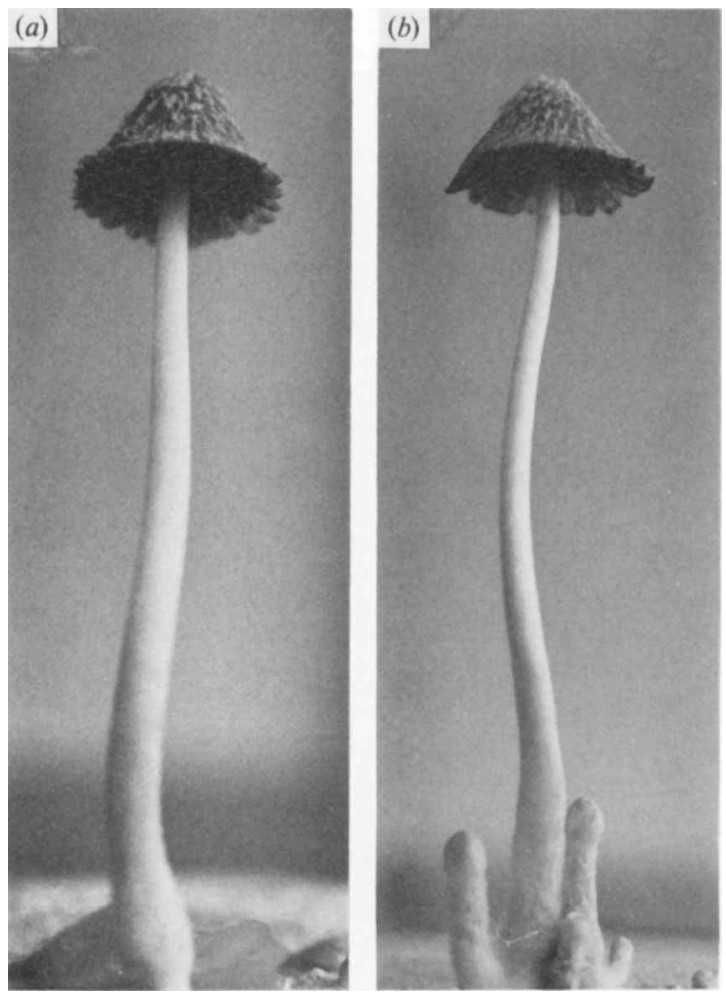

Fig. 1. Fruit bodies of dikaryotic and Amut Bmut cultures. (a) Dikaryon $(A 2 B 2 \times A 7 B 7) ;(b)$ Amut Bmut-1.

Table 3. Results of crosses between Amut and Bmut strains, and Amut Bmut and wild-type strains

Cross*

Amut $B 8 \times A 7$ Bmut

Amut $B 2 \times A 2$ Bmut

Amut Bmut-1 $\times$ A7 B7

Amut Bmut-4 $\times$ A8 B8
Segregation of $A$ and $B$ factors

$\begin{array}{lll}23 \text { Amut B8 } & 24 A 7 \text { Bmut } & 20 A 7 B 8 \\ 20 \text { Amut B2 } & 19 A 2 B \text { Bmut } & 16 A 2 B 2 \\ 23 \text { Amut B7 } & 21 A 7 B m u t & 27 A 7 B 7 \\ 18 \text { Amut B8 } & 16 \text { A B Bmut } & 20 A 8 B 8\end{array}$

* The Amut Bmut strains were selected from those recovered from crosses between $A m u t$ and Bmut.

action of both $A$ and $B$ factors. The $A$ factor regulates initiation of clamp cells, while the $B$ factor regulates their fusion to subapical cells. In the $B m u t$ strains this part of the $B$-dependent event was not evident on account of $A$-dependent sequences being 'off'. The presence of a large number of true clamps in the Amut Bmut strains, however, indicated that the $B$ genes responsible for clamp cell fusion had been switched on.

A study of basidiospore formation in the Amut Bmut strains was carried out by dissecting portions of gills from young fruit bodies formed on Amut Bmut mycelia. These were then observed under a fluorescent microscope. The meiotic process and formation of four spores on the basidium in Amut Bmut fruit bodies (Fig. 2) were similar to those observed in fruit bodies of dikaryotic strains reported previously (Miyake et al., 1980).

Mimicry of the normal dikaryon by the Amut Bmut strains was, however, not absolute. Irregularities were observed in certain features including the extent of true clamps formed as compared to normal dikaryons. Several pseudoclamps were intermingled with true clamps and the ratio of pseudoclamps to true clamps varied. Mycelial preparations of Amut Bmut strains 

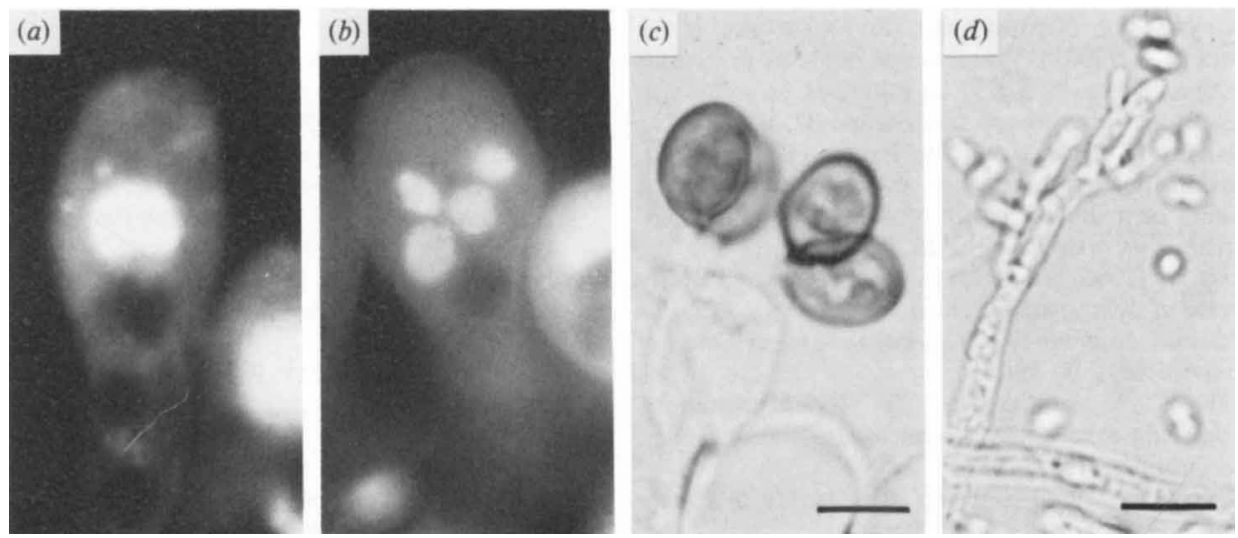

Fig. 2. Photomicrographs showing the representative stages of sporulation and an oidiophore in Amut Bmut-1. (a) Meiotic prophase I ; (b) tetrad nuclei; (c) tetrad basidiospores; (d) oidiophore. Basidia $(a, b)$ were stained with propidium iodide. Photographs were taken under phase contrast $(c, d)$ and fluorescence $(a, b)$ microscopes. Bar, $1.0 \mu \mathrm{m}(a, b, c)$ and $10 \mu \mathrm{m}(d)$.

also revealed the presence of a considerable number of oidia (Table 2). The oidia were all uninucleate and are shown in Fig. 2 along with an oidiophore formed by Amut Bmut mycelia. Although dikaryotic strains produced oidia the number per culture was relatively much smaller than that produced by the monokaryotic and Amut Bmut strains (Table 2). To test whether the oidia produced by Amut Bmut mutants possessed the potential to grow into self-fertile Amut Bmut strains, single oidium isolations were carried out. All of the isolates grew into strains which were dikaryotic in appearance and fertile. These findings suggest that in Coprinus the asexual morphogenetic pathways and sexual morphogenetic pathways controlled by incompatibility factors operate independently and are not inhibited by one another.

The meiotic events and formation of four spores on the basidium of Amut Bmut fruit bodies resembled those observed in homothallic species, where fertile dikaryotic mycelia are formed in the absence of fusion of two compatible monokaryotic mycelia (Harder, 1926). The formation of fruit bodies by such mutant strains of heterothallic species without mating might represent a possible pathway in evolution by which homothallic species evolve from heterothallic species of Basidiomycetes.

S. S. gratefully acknowledges the receipt of a research fellowship from the Ministry of Education, Science and Culture, Japan. This work has been supported by a grant-in-aid for special project research from the Ministry of Education, Science and Culture of Japan (no. 5811900).

\section{REFERENCES}

Casselton, L. A. (1978). Dikaryon formation in the higher basidiomycetes. In The Filamentous Fungi, vol. 3, pp. 278-297. Edited by J. E. Smith \& D. R. Berry. London: Arnold.

DAY, P. R. (1963). Mutations of the $A$ mating type factor in Coprinus lagopus. Genetical Research 4, 5564.

FrIES, L. (1953). Factors promoting growth of Coprinus fimetarius (L.) under high temperature conditions. Physiologia plantarum 6, 551-563.

HARDER, R. (1926). Mikrochirurgische Untersuchungen über die geschlechtliche Tendenz der Paarkerne des homothallischen Coprinus sterquilinus Fries. Planta 2, 263-275.

haylock, R. W., Economou, A. \& Casselton, L. A.
(1980). Dikaryon formation in Coprinus cinereus: selection and identification of $B$ factor mutants. Journal of General Microbiology 121, 17-26.

Koltin, Y. (1970). Development of Amut Bmut strain of Schizophyllum commune. Archiv für Mikrobiologie 74, 123-128.

Koltin, Y. (1978). Genetic structure of incompatibility factors - the $\mathrm{ABC}$ of sex. In Genetics and Morphogenesis in the Basidiomycetes, pp. 31-54. Edited by M. N. Schwalb \& P. G. Miles. New York: Academic Press.

Koltin, Y., Stamberg, J. \& Lemke, P. A. (1972). Genetic structure and evolution of the incompatibility factors in higher fungi. Bacteriological Reviews 36, 156-171. 
Kuroiwa, T., Nishibayashi, S., Kawano, S. \& SUZUKI, T. (1981). Visualization of DNA in various phages $(T 4, \chi, T 7, \phi 29)$ by ethidium bromide epifluorescent microscopy. Experientia 37, 969-970.

Miyake, H., Tanaka, K. \& Ishikawa, T. (1980). Basidiospore formation in monokaryotic fruiting bodies of a mutant strain of Coprinus macrorhizus. Archives of Microbiology 126, 207-212.

PaRaG, Y. (1962). Mutations in the $B$ incompatibility factor of Schizophyllum commune. Proceedings of the National Academy of Sciences of the United States of America 48, 743-750.

RAPER, C. A. \& RAPER, J. R. (1966). Mutations modifying sexual morphogenesis in Schizophyllum. Genetics 54, 1151-1168.

RAPER, J. R. (1966). Genetics of Sexuality in Higher Fungi. New York: Ronald Press.

RAPER, J. R. \& SAN ANtonio, J. P. (1954). Hetero- karyotic mutagenesis in hymenomycetes. 1. Heterokaryosis in Schizophyllum commune. American Journal of Botany 41, 69-86.

Raper, J. R., Boyd, D. H. \& Raper, C. A. (1965). Primary and secondary mutations at the incompatibility loci in Schizophyllum. Proceedings of the National Academy of Sciences of the United States of America 53, 1324-1332.

SwiezYNSKI, K. M. \& DAY, P. R. (1960a). Heterokaryon formation in Coprinus lagopus. Genetical Research 1, 114-128.

SwiEZYNSKI, K. M. \& DAY, P. R. (1960b). Migration of nuclei in Coprinus lagopus. Genetical Research 1, 129 139.

Uno, I. \& Ishikawa, T. (1971). Chemical and genetical control of induction of monokaryotic fruiting bodies in Coprinus macrorhizus. Molecular and General Genetics 113, 228-239. 